\title{
Altered gray matter volume in patients with herpes zoster and postherpetic neuralgia
}

This article was published in the following Dove Medical Press journal: Journal of Pain Research

\author{
Jiaqi Liu',* \\ Lili $\mathrm{Gu}^{2, *}$ \\ Qing Huang' \\ Shunda Hong' \\ Xianjun Zeng' \\ Daying Zhang ${ }^{2}$ \\ Fuqing Zhou' \\ Jian Jiang'
}

'Department of Radiology,

${ }^{2}$ Department of Pain, The First

Affiliated Hospital, Nanchang

University, Nanchang, Jiangxi, People's

Republic of China

*These authors contributed equally to this work
Correspondence: Jian Jiang Department of Radiology, The First Affiliated Hospital, Nanchang University, 17 Yongwaizheng Street, Nanchang 330006, Jiangxi, People's Republic of China

Tel +8679188693825

Email jij2002cn@।26.com
Purpose: The aim of this study was to measure brain alterations in patients with herpes zoster (HZ) and postherpetic neuralgia (PHN) and compare their differences using a voxel-based morphometry (VBM) technique.

Materials and methods: Thirty-three patients with HZ, 22 patients with $\mathrm{PHN}$, and 28 well-matched healthy controls (HCs) were recruited. Magnetic resonance imaging data were acquired for all subjects and analyzed using the VBM method. The changes in gray matter volume (GMV) in HZ and PHN groups were compared with those in HC group, and the GMV differences were also compared between the PHN and HZ groups. Further correlation analysis and receiver operating characteristic curves were used to confirm the significance of GMV changes in various brain regions.

Results: Compared with HCs, decreased GMV was found in the bilateral insular lobes and increased GMV was found in the bilateral thalamus in the HZ group. In the PHN group, GMV decreased in the bilateral insula lobes, right middle frontal gyrus, bilateral precentral gyrus, and left postcentral gyrus and increased in the left cerebellar posterior lobe, right parahippocampal gyrus, and right lentiform nucleus. In addition, the PHN group exhibited increased GMV in the left cerebellar tonsil, culmen, and left lentiform nucleus and decreased GMV in the right precentral gyrus compared with the HZ group. Further correlation analysis and receiver operating characteristic curves revalidate the significance of most of these abnormal brain regions. Conclusion: The VBM method revealed widespread GMV abnormalities in HZ and PHN patients. The brains of PHN patients have broader abnormalities in nonpain-related regions, suggesting the complexity of a central mechanism. When PHN patients were compared with $\mathrm{HZ}$ patients, the left cerebellar tonsil, culmen, and left lentiform nucleus corresponded to greater area under the curve, suggesting that abnormalities in these regions are risk factors for $\mathrm{HZ}$ patients' transformation to $\mathrm{PHN}$.

Keywords: chronic pain, neuroimaging, ROC, fMRI, VBM

\section{Introduction}

Herpes zoster $(\mathrm{HZ})$ is an infection characterized by reactivation of varicella-zoster virus. It can result in erythema, clustered shingles, and neuralgia. Postherpetic neuralgia (PHN) is the most common complication of $\mathrm{HZ}^{1}$ and is defined as typical chronic neuropathic pain that persists for more than 1 month after the zoster is cured. ${ }^{2} \mathrm{PHN}$ is clinically characterized by extreme pain, usually accompanied with various abnormal sensory symptoms, ${ }^{3,4}$ PHN negatively affects patients' quality of life and mental health, and PHN patients are at a particularly high risk of developing depression and anxiety. ${ }^{5,6}$ Moreover, PHN is becoming an increasing economic burden given the current 
aging society. Complexity of the PHN mechanism results in unsatisfactory management of PHN.

With the development of neuroimaging, functional magnetic resonance imaging (fMRI) has contributed to a deeper understanding of the central nervous system and neuropathic pain. Resting-state fMRI (rs-fMRI) focuses on the spontaneous activity of the brain in the conscious resting state. It is a noninvasive, radiation-free method for exploring the neural mechanisms of neuropathic pain and is widely used in PHN. Although growing evidence shows that the brain is involved in pain regulation and pain defense, ${ }^{2,7}$ the central mechanism of PHN is not yet fully understood. Neuroimaging studies have revealed abnormal activity in the brains of PHN patients, including in the insula, thalamus, cerebellum, limbic system, prefrontal lobe, and temporal lobe, using regional homogeneity (ReHo) and fractional aptitude of lowfrequency fluctuations, which are commonly used methods in rs-fMRI. ${ }^{8-11}$ In addition, the application of rs-fMRI is not limited to locating certain brain regions but also in complex brain networks. More recently, voxel-mirrored homotopic connectivity and seed-based functional connectivity analyses have revealed decreased homotopic connectivity in the dorsolateral prefrontal cortex and the precuneus and posterior cingulate cortex, indicating disrupted intrinsic connectivity between these regions and the default-mode network (DMN) in PHN brains. ${ }^{12}$ Utilizing the arterial spin labeling technique to choose "seeds" highly correlated with pain intensity and calculating the functional connectivity showed that reward circuitry, especially the striatum, thalamus, and insula, was highly correlated with pain in PHN patients. ${ }^{13}$

Structure is the basis of function, although less is known about the structural changes of the PHN brain because few studies have been conducted on this topic. Previous studies have used diffusion tensor imaging (DTI), diffusional kurtosis imaging (DKI), or voxel-based morphometry (VBM) and have independently reported that PHN is closely associated with structural pathological changes in the brain. ${ }^{14-16}$ These results largely overlap with brain regions that show abnormal activity, such as the insula, cerebellum, frontal lobe, and temporal lobe. In the 1990s, using light microscopy, researchers found that similar to the skin healing with scars due to shingles, the peripheral nervous system and the central nervous system can also exhibit scars upon healing. ${ }^{17,18}$ These changes lead to a series of neurological functional changes, followed by progression to PHN. Therefore, to understand the central mechanism of PHN, identifying the functional and structural changes in the central nervous system of patients with $\mathrm{HZ}$ is also important. Hong et al ${ }^{19}$ explored connectivity network disruptions and found DMN damage in both $\mathrm{HZ}$ and PHN patients. However, the differences in gray matter (GM) alterations in $\mathrm{HZ}$ and $\mathrm{PHN}$ patients are not clear. Thus, we hypothesized that $\mathrm{HZ}$ and PHN both alter the gray matter volume (GMV) of the brain, which in turn affects the patients' clinical status. VBM is an objective method that enables a voxel-wise estimation of brain changes or direct estimation of the local amount of a specific tissue via original data analysis. Moreover, VBM can quantitatively detect GM differences without requiring a prior definition of the region of interest, thus avoiding the subjective influence of researchers. As VBM is automatic, comprehensive, objective, and repeatable, among other advantages, it has been widely used to analyze many diseases associated with brain morphological abnormalities, such as schizophrenia, Alzheimer's disease, and stroke. This study was designed to measure and compare the morphological changes of the brain in patients with $\mathrm{HZ}$ and PHN using the VBM method and to analyze the correlation between significant brain regions and clinical indexes, such as pain degree and pain duration, to provide imaging evidence for further demonstration of the pathogenesis of PHN pain.

\section{Materials and methods Participants}

Fifty-five right-handed patients with $\mathrm{HZ}$ and $\mathrm{PHN}$ were recruited from the Pain Department of the First Affiliated Hospital of Nanchang University, and two consultant physicians from the Pain Department made the clinical diagnoses. The intensity levels of spontaneous pain were assessed using a visual analog scale (VAS) $)^{20,21}$ ranging from 0 (no pain) to 10 (highest intolerable pain) before fMRI scanning. Twentytwo PHN patients were diagnosed, based on the International Association for the Study of Pain, with the presence of pain persisting for $>1$ month (1-20 months, mean 3 months) after recovery from $\mathrm{HZ}$ infection and with a pain intensity of at least 5 on the VAS. The age of the patients with PHN ranged from 54 to 78 years. In PHN patients, skin lesions were localized on the left side of the body in 13 cases and on the right side in 9 cases. The recruitment criteria for the 33 patients with $\mathrm{HZ}$ were exhibiting shingles within the last 1 month that did not subside and a VAS score of 5 or more. The ages of the patients with $\mathrm{HZ}$ ranged from 37 to 73 years. The skin lesions of the $\mathrm{HZ}$ patients were localized on the left side of the body in 16 cases and on the right side in 17 cases. Patients who had other pain disorders were excluded, and none of the included patients had a history of any other psychiatric illness or neurological illness, head injury, or alcohol or drug abuse. 
Using advertisements posted on the department bulletin board and in a WeChat circle of friends, we recruited 28 righthanded healthy controls (HCs) age- and sex-matched with the $\mathrm{HZ}$ and PHN patients. The HCs ranged in age from 38 to 72 years. None of the HCs had any spontaneous pain or sensory abnormalities, substance abuse, neurological disorders, or significant psychiatric disorders. The healthy subjects also had no family history of mental disorders.

Written informed consent was obtained from all participants or participants' guardians before data acquisition. The current study was approved by the Medical Research Ethics Committee and the institutional Review Board of The First Affiliated Hospital of Nanchang University and in accordance with the principles of the Declaration of Helsinki.

\section{Image acquisition}

All MRI experiments were implemented using a 3.0 T Siemens TrioTIM Scanner (Erlangen, Bavaria, Germany) at the Radiology Department, the First Affiliated Hospital of Nanchang University. Each participant underwent a threedimensional, high-resolution T1-weighted structural MRI scan by a magnetization-prepared rapidly acquired gradient echo sequence with the following parameters: repetition time $=1,900 \mathrm{~ms}$, echo time $=2.26 \mathrm{~ms}$, flip angle $=9^{\circ}$, matrix $=240 \times 256$, field of view $=215 \times 230 \mathrm{~mm}$, slice thickness $=1.0$ $\mathrm{mm}$, slice gap $=0 \mathrm{~mm}$, sweep time $=8$ minutes, and 176 sagittal slices. In the MRI scans, participants were instructed to close their eyes but remain awake and to keep their mind clear, and earplugs were used to reduce the machine's noise. After the scan, the subjects were asked whether they stayed awake during the entire scan. In addition, all subjects underwent routine conventional T2-weighted scans to exclude visible brain structural abnormalities.

\section{Data preprocessing}

The rs-fMRI data processing assistant (DPARSF - V2.1, http://rfmri.org/DPARSF) toolkit based on the MATLAB (MathWorks, Natick, MA, USA) platform was used for data processing and statistical analysis of high-resolution 3D-T1WI images. The general steps were as follows: 1) T1WI images were observed for quality to exclude poor quality images (no images were excluded from this study). 2) Images were reformatted. 3) A common template was created by group average and all original images registering to the common template by affine transformation. 4) The T1-weighted images were corrected for inhomogeneities and classified into GM, white matter, and cerebrospinal fluid. 5) The GM segment is then spatially normalized (linear and nonlinear normalizations and Jacobian determinants) to match Montreal Neurological Institute (MNI) space. ${ }^{22}$ The orientation is determined from unit vectors directed along $X, Y$, and $Z$ brain axes. 6) The GM segment was resliced to a size of $1.5 \times 1.5 \times 1.5 \mathrm{~cm}^{3}$ and smoothed with a $3-\mathrm{mm}$ full-width at half-maximum isotropic Gaussian kernel. 7) $Z$-standardization for better comparison.

\section{Statistical analyses}

The VBM data of the $\mathrm{HZ}$ and PHN groups were separately compared with the HC group by two-sample $t$-tests using REST software on the MATLAB platform (two-tailed, voxellevel $P<0.01$; Gaussian random field correction, cluster-level $P<0.05)$. Age and gender were used as covariates. The MNI coordinates of the significant regions were recorded, and the statistically significant voxels were superimposed on the group-standard 3D-T1WI image to generate a pseudocolor image. The statistically significant brain regions of the PHN and $\mathrm{HZ}$ groups were extracted as regions of interest, and their correlations with the respective VAS and disease duration were analyzed using IBM SPSS 22.0 (Chicago, IL, USA) software; $P<0.05$ was considered significant. Receiver operating characteristic (ROC) curves were also analyzed using SPSS 22.0 to evaluate diagnoses of significant brain regions, and Bonferroni corrections were used for all multiple comparisons. SPSS 22.0 software was also used to examine the differences in age among the three groups through nonparametric statistical test. Gender was compared between the two case groups and the HC group using the chi-squared test. $P<0.05$ indicated that the difference was statistically significant.

\section{Results}

\section{Demographic and clinical characteristics}

The general clinical characteristics of the HZ and PHN patients are listed in Table 1. The HZ and PHN groups did not differ significantly in age $(P=0.22,0.96,0.19$ for $\mathrm{HZ}$ vs $\mathrm{HC}$, PHN vs HZ, PHN vs HZ, respectively, $t$-test) or gender ( $P=0.52,0.56,0.91$ for $\mathrm{HZ}$ vs HC, PHN vs HZ, PHN vs HZ, respectively, chi-squared test) from the $\mathrm{HC}$ group.

\section{GMV alterations in the $\mathrm{HZ}$ and $\mathrm{PHN}$ patients}

Compared with the $\mathrm{HC}$ group, the bilateral insula exhibited a significantly reduced $(P<0.01)$ GMV (within right Rolandic Operculum), and the bilateral thalamus exhibited a significantly increased GMV $(P<0.01)$ in the HZ group (Figure 1, Table 2).

In the PHN group, the left insula lobe, right middle frontal gyrus (MFG), right insula lobe, left precentral 
Table I Demographic and clinical characteristics of the participants

\begin{tabular}{|c|c|c|c|c|}
\hline & & $\mathrm{HZ}$ patients & PHN patients & Healthy controls \\
\hline Age (years, $M \pm I Q R)$ & & $57.00 \pm 12.50$ & $65.50 \pm 11.25$ & $54.00 \pm 9.75$ \\
\hline Gender (male:female) & & $13 / 20$ & $9 / 13$ & $9 / 19$ \\
\hline Skin lesions (L:R) & & $16 / 17$ & $13 / 9$ & - \\
\hline \multirow[t]{3}{*}{ Subregions } & $\begin{array}{l}\text { Head and neck } \\
(L: R)\end{array}$ & $5 / 4$ & $0 / 2$ & - \\
\hline & $\begin{array}{l}\text { Upper body } \\
(\mathrm{L}: \mathrm{R})\end{array}$ & $9 / 4$ & $8 / 5$ & - \\
\hline & $\begin{array}{l}\text { Lower body } \\
(L: R)\end{array}$ & $2 / 9$ & $5 / 2$ & - \\
\hline Pain duration $(M \pm I Q R)$ & & $9.00 \pm 11.50$ (days) & $2.00 \pm 2.25$ (months) & - \\
\hline Shingle duration $(M \pm I Q R)$ & & $8.00 \pm 10.50$ (days) & $1 \mathrm{I} .50 \pm 9.50$ (days) & - \\
\hline VAS score $(M \pm I Q R)$ & & $6.00 \pm 2.00$ & $6.50 \pm 1.00$ & - \\
\hline
\end{tabular}

Abbreviations: $\mathrm{HZ}$, herpes zoster; L, left; M, median; PHN, postherpetic neuralgia; R, right; VAS, visual analog scale.

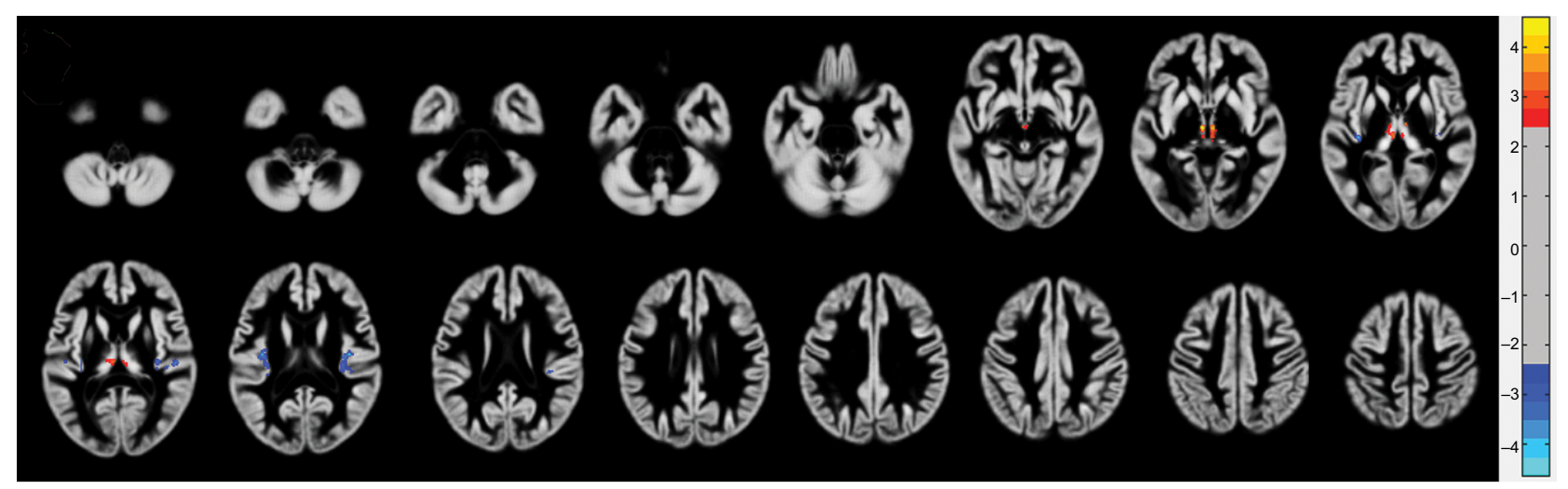

Figure I Local GMV difference between $\mathrm{HZ}$ patients and $\mathrm{HC}$.

Note: The warm colors indicate increased GMV, and cool colors indicate decreased GMV.

Abbreviations: GMV, gray matter volume; $\mathrm{HC}$, healthy control; $\mathrm{HZ}$, herpes zoster.

Table 2 Significant differences in GMV between the HZ and HC groups

\begin{tabular}{|c|c|c|c|c|c|c|c|}
\hline \multirow[t]{2}{*}{ Brain area } & \multirow{2}{*}{$\begin{array}{l}\text { L: left } \\
\text { R: right }\end{array}$} & \multirow{2}{*}{$\begin{array}{l}\text { Brodmann's } \\
\text { area }\end{array}$} & \multicolumn{3}{|l|}{ MNI } & \multirow{2}{*}{$\begin{array}{l}\text { Peak } \\
\text { intensity }\end{array}$} & \multirow{2}{*}{$\begin{array}{l}\text { Number } \\
\text { of voxels }\end{array}$} \\
\hline & & & $x$ & $Y$ & $\mathbf{Z}$ & & \\
\hline \multicolumn{8}{|l|}{ Brain areas of decreased GMV } \\
\hline Insula & L & $\mathrm{BA} / 3$ & -34.5 & -22.5 & 6 & -3.892 & 316 \\
\hline Insula & $\mathrm{R}$ & $\mathrm{BA} \mid 3$ & 40.5 & -13.5 & 16.5 & -3.924 & 379 \\
\hline Rolandic_Operculum & $\mathrm{R}$ & $\mathrm{BA} / 3$ & 40.5 & -13.5 & 16.5 & -3.924 & 379 \\
\hline \multicolumn{8}{|l|}{ Brain areas of increased GMV } \\
\hline Thalamus & - & - & -4.5 & -13.5 & -1.5 & 4.31 & 395 \\
\hline
\end{tabular}

Abbreviations: GMV, gray matter volume; HC, healthy control; $\mathrm{HZ}$, herpes zoster; MNI, Montreal Neurological Institute.

gyrus, right precentral gyrus, and left postcentral gyrus exhibited a significant decreased GMV $(P<0.01)$, and the left cerebellar posterior lobe (CPL), right parahippocampal gyrus, and right lentiform nucleus exhibited a significant increased GMV $(P<0.01)$ compared with the HC group (Figure 2, Table 3).

Compared with the HZ group, the PHN group showed a significantly increased GMV $(P<0.01)$ in the left cerebellar tonsil, culmen (a part of vermis), and left lentiform nucleus and a significantly decreased GMV $(P<0.01)$ in the right precentral gyrus (Figure 3, Table 4).

\section{Clinical correlations of GMV in different periods of $\mathrm{HZ}$ and $\mathrm{PHN}$ patients}

The GMV of the thalamus was positively correlated with VAS scores $(P=0.01, r=0.443)$ in HZ patients (Figure 4). 


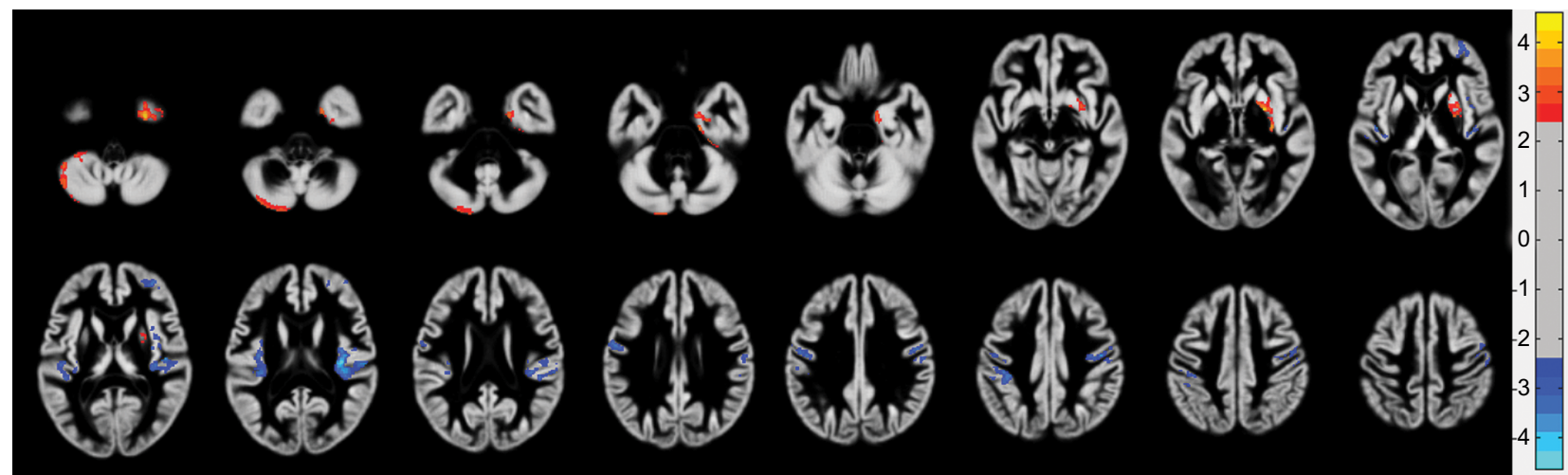

Figure 2 Local GMV difference between PHN and HC patients.

Note: The warm colors indicate increased GMV, and the cool colors indicate decreased GMV.

Abbreviations: GMV, gray matter volume; HC, healthy control; PHN, postherpetic neuralgia.

Table 3 Significant differences in GMV between the PHN and HC groups

\begin{tabular}{|c|c|c|c|c|c|c|c|}
\hline \multirow[t]{2}{*}{ Brain area } & \multirow{2}{*}{$\begin{array}{l}\text { L: left } \\
\text { R: right }\end{array}$} & \multirow{2}{*}{$\begin{array}{l}\text { Brodmann's } \\
\text { area }\end{array}$} & \multicolumn{3}{|l|}{ MNI } & \multirow{2}{*}{$\begin{array}{l}\text { Peak } \\
\text { intensity }\end{array}$} & \multirow{2}{*}{$\begin{array}{l}\text { Number } \\
\text { of voxels }\end{array}$} \\
\hline & & & $x$ & $\boldsymbol{Y}$ & $\mathbf{Z}$ & & \\
\hline \multicolumn{8}{|l|}{ Brain areas of decreased GMV } \\
\hline Insula & L & BAI3 & -46.5 & -25.5 & 13.5 & -4.599 & 665 \\
\hline Middle frontal gyrus & $R$ & BAIO & 36 & 51 & 20.5 & -4.034 & 326 \\
\hline Insula & R & $\mathrm{BA} \mid 3$ & 36 & -19.5 & 18 & -6.278 & 1,436 \\
\hline Precentral gyrus & L & BA6 & -55.5 & -6 & 27 & -4.835 & 364 \\
\hline Precentral gyrus & $\mathrm{R}$ & BA4 & 46.5 & -13.5 & 40.5 & -4.385 & 631 \\
\hline Postcentral gyrus & L & BA40 & -37.5 & -33 & 40.5 & -4.080 & 402 \\
\hline \multicolumn{8}{|l|}{ Brain areas of increased GMV } \\
\hline Cerebellum posterior lobe & L & - & -22.5 & -33 & -46.5 & 4.106 & $\mathrm{I}, 103$ \\
\hline \multirow[t]{2}{*}{ Parahippocampal gyrus } & $\mathrm{R}$ & BA28 & 16.5 & -6 & -28.5 & 5.005 & 640 \\
\hline & & BA35 & & & & & \\
\hline Lentiform nucleus & $\mathrm{R}$ & - & 24 & 1.5 & -1.5 & 5.587 & 560 \\
\hline
\end{tabular}

Abbreviations: GMV, gray matter volume; HC, healthy control; MNI, Montreal Neurological Institute; PHN, postherpetic neuralgia.

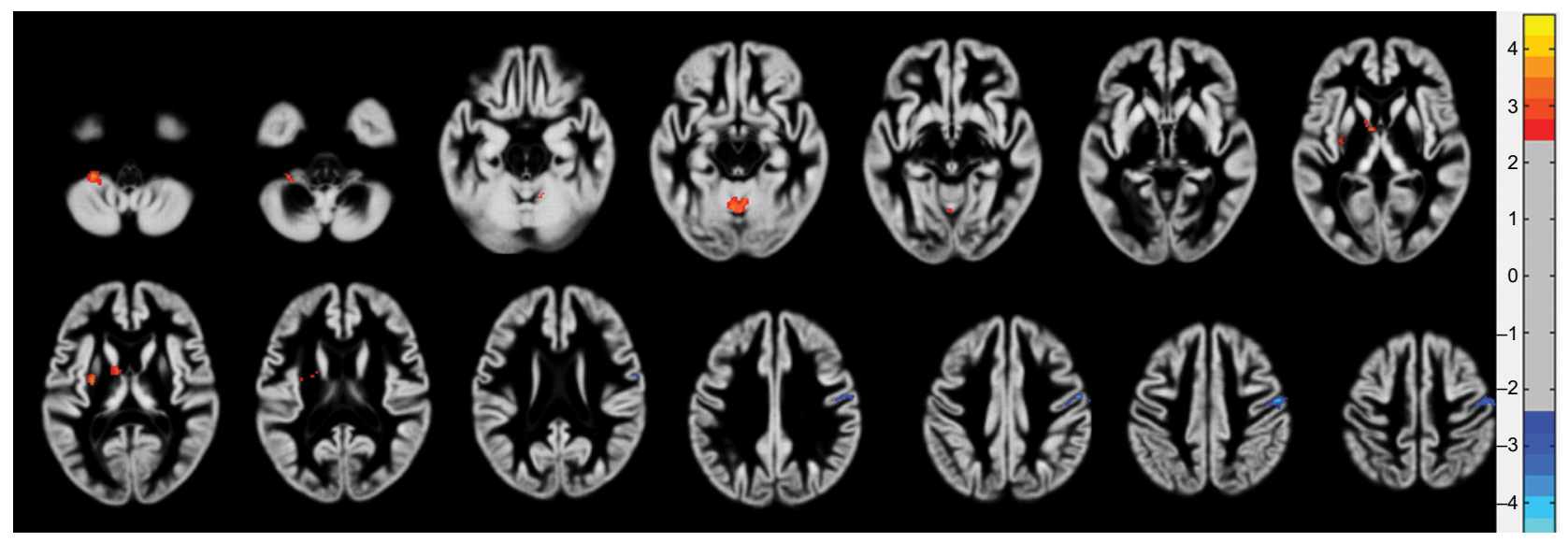

Figure 3 Local GMV difference between PHN and $\mathrm{HZ}$ patients.

Note: The warm colors indicate increased GMV, and the cool colors indicate decreased GMV.

Abbreviations: GMV, gray matter volume; $\mathrm{HZ}$, herpes zoster; $\mathrm{PHN}$, postherpetic neuralgia.

In PHN brains, the changes in GMV in the left CPL and right parahippocampal gyrus were positively correlated with VAS scores $(P=0.039, r=0.444$ and $P=0.017, r=0.503$; Figure 5A,B). The change in GMV in the left insula was negatively correlated with pain duration $(P=0.016, r=-0.509$; Figure $5 \mathrm{C})$. The GMV of the left precentral gyrus and left postcentral gyrus showed significant negative correlations with VAS scores $(P=0.019, r=-0.497$ and $P=0.015, r=-0.516$ ) in PHN subjects (Figure 5D,E). 
Table 4 Significant differences in GMV between the PHN and $\mathrm{HZ}$ groups

\begin{tabular}{|c|c|c|c|c|c|c|c|}
\hline \multirow[t]{2}{*}{ Brain area } & \multirow{2}{*}{$\begin{array}{l}\text { L: left } \\
\text { R: right }\end{array}$} & \multirow{2}{*}{$\begin{array}{l}\text { Brodmann's } \\
\text { area }\end{array}$} & \multicolumn{3}{|l|}{ MNI } & \multirow{2}{*}{$\begin{array}{l}\text { Peak } \\
\text { intensity }\end{array}$} & \multirow{2}{*}{$\begin{array}{l}\text { Number } \\
\text { of voxels }\end{array}$} \\
\hline & & & $x$ & $Y$ & $\mathbf{Z}$ & & \\
\hline $\begin{array}{l}\text { Brain areas of decreased GMV } \\
\text { Precentral gyrus }\end{array}$ & $\mathrm{R}$ & BA3,6 & 54 & -12 & 48 & -4.596 & 447 \\
\hline Brain areas of increased GMV & & & & & & & \\
\hline Culmen & - & - & -3 & -57 & -10.5 & 4.467 & 368 \\
\hline Cerebellar tonsil & L & - & -28.5 & -39 & -46.5 & 4.381 & 275 \\
\hline Lentiform nucleus & L & - & -28.5 & -7.5 & 12 & 4.725 & 344 \\
\hline
\end{tabular}

Abbreviations: GMV, gray matter volume; HZ, herpes zoster; MNI, Montreal Neurological Institute; PHN, postherpetic neuralgia.
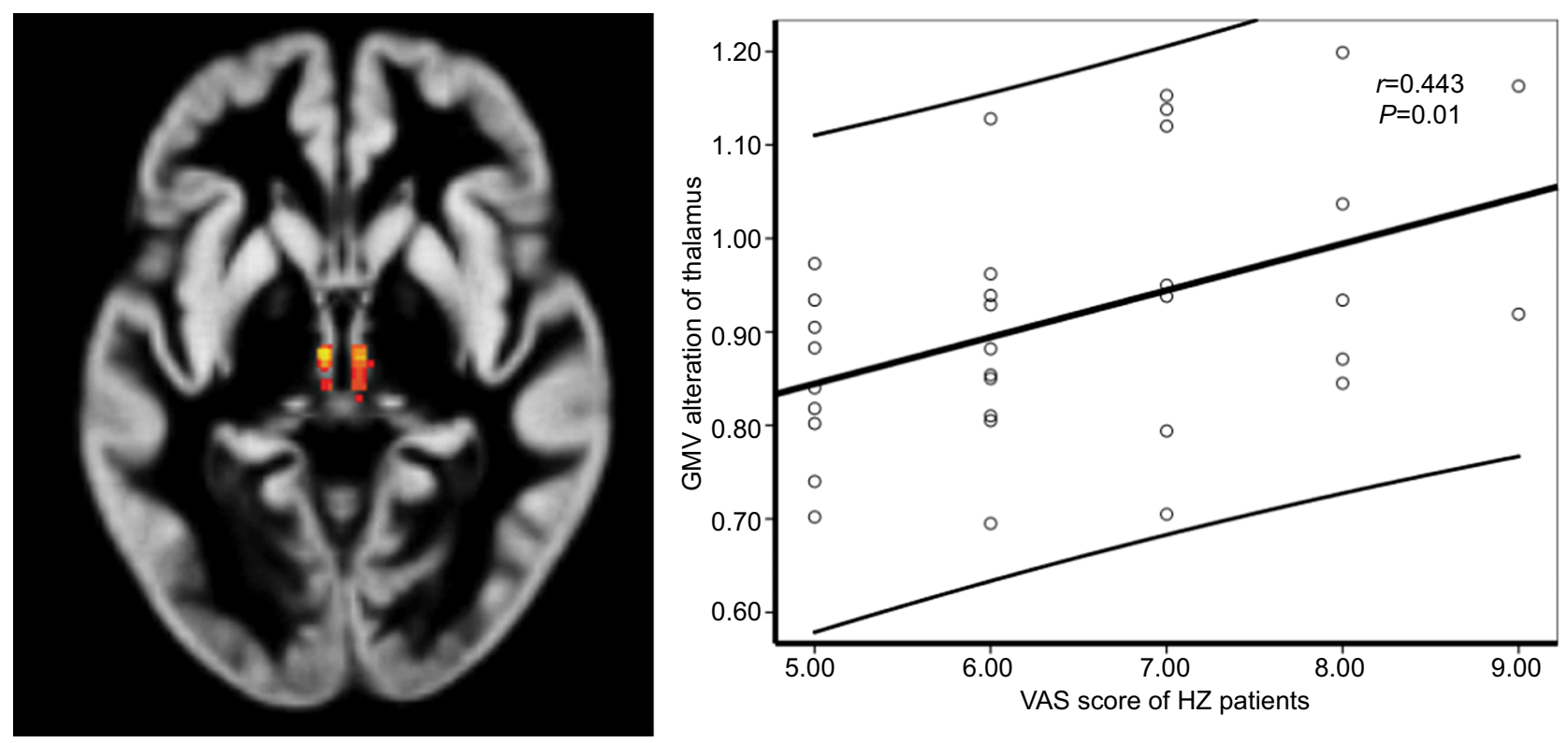

Figure $4 \mathrm{~A}$ significant correlation between GMV in the bilateral thalamus and VAS scores in $\mathrm{HZ}$ patients.

Abbreviations: GMV, gray matter volume; $\mathrm{HZ}$, herpes zoster; VAS, visual analog scale.

The brain regions with significantly altered GMV were not correlated with the VAS scores or duration when comparing the PHN and $\mathrm{HZ}$ groups $(P>0.05)$.

\section{ROC analysis for discriminating $\mathrm{HZ}$ and $\mathrm{PHN}$ patients}

ROC analyses revealed excellent sensitivity and specificity with satisfactory accuracy for discriminant function. The area under the curve (AUC) values in bilateral insula (AUC $=0.821$ for right insula and 0.835 for left insula) were relatively high, which showed reliable classification to discriminate $\mathrm{HZ}$ patients from HCs (Figure 6, Table 5). The AUC values in bilateral insula ( $\mathrm{AUC}=0.938$ for right insula and 0.890 for left insula), bilateral precentral gyrus ( $A U C=0.821$ for right precentral gyrus and 0.835 for left precentral gyrus), right MFG (AUC $=0.885)$, and left postcentral gyrus $(\mathrm{AUC}=0.881)$ were relatively high, which showed reliable classification to discriminate PHN patients from HCs (Figure 7, Table 6). In addition, left cerebellar tonsil (AUC $=0.805)$, culmen (AUC $=0.788)$, and left lentiform nucleus (AUC $=0.826$ ) also demonstrated higher AUC for excellent discriminant function between PHN and $\mathrm{HZ}$ patients (Figure 8, Table 7).

\section{Discussion}

In this study, VBM was used to investigate and compare alterations in GMV in $\mathrm{HZ}$ and PHN patients. Both $\mathrm{PHN}$ and $\mathrm{HZ}$ patients showed extensive and significant alterations in GMV. PHN has extreme effects on the brain. In addition to regions associated with pain, regions related to emotion (lentiform nucleus), memory (parahippocampus), and somatomotor functions (precentral gyrus) also showed significant changes in GMV. These results indicated that brain regions related to emotion, memory, and motor function, in addition to pain-related brain regions, are also involved in the cen- 
A

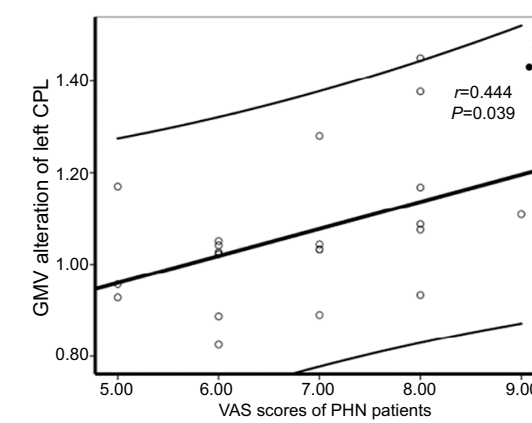

C

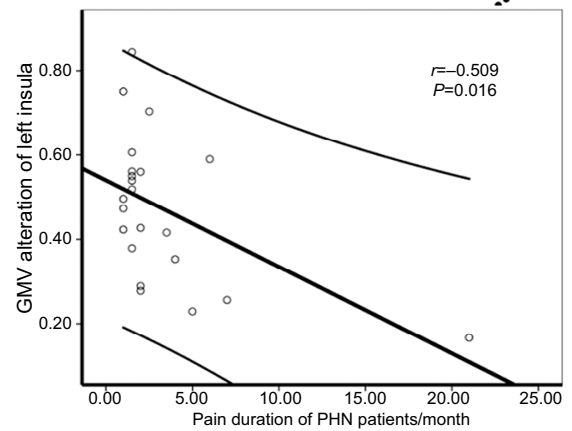

B

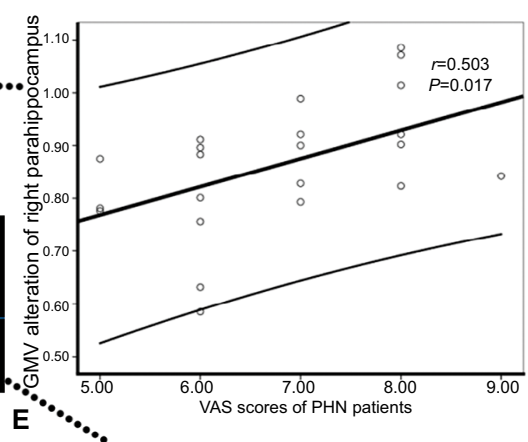

D
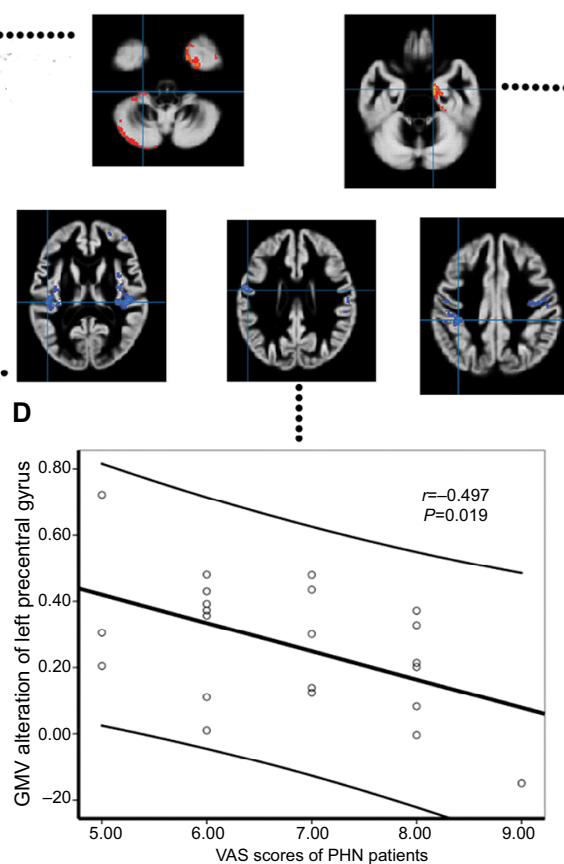

E $\bullet \cdot \bullet$

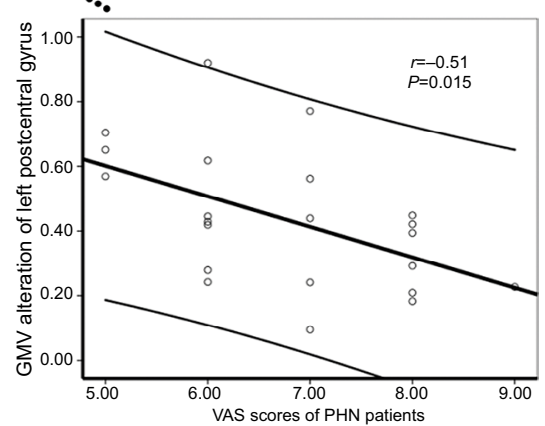

Figure 5 There were significant correlations between GMV alteration in the left CPL (A) and right parahippocampus (B) with VAS scores in PHN patients. Decreased GMV alteration of the left insula, with pain duration (C). Decreased GMV alteration in left precentral gyrus (D), and left postcentral gyrus (E), with VAS scores in PHN patients. Abbreviations: CPL, cerebellar posterior lobe; GMV, gray matter volume; PHN, postherpetic neuralgia; VAS, visual analog scale.

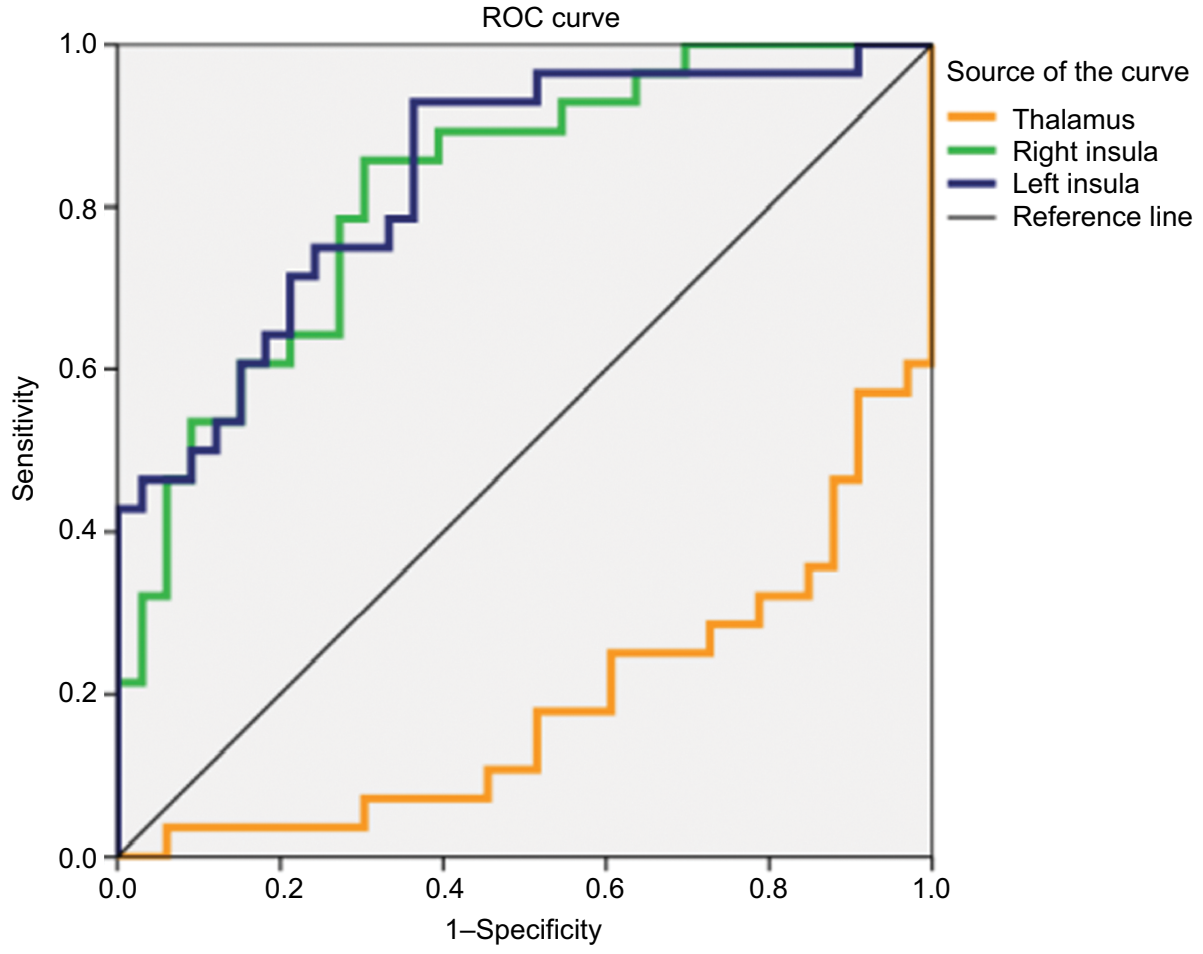

Figure 6 ROC curves of GMV in the altered regions for distinguishing $\mathrm{HZ}$ patients from $\mathrm{HCs}$.

Abbreviations: GMV, gray matter volume; HCs, healthy controls; HZ, herpes zoster; ROC, receiver operating characteristic. 
Table 5 ROC analysis for differentiating the $\mathrm{HZ}$ and $\mathrm{HC}$ groups

\begin{tabular}{|l|l|l|l|l|l|l|}
\hline Brain region & AUC & Cutoff point & Sensitivity (\%) & Specificity (\%) & \multicolumn{2}{|l|}{ 95\% Cl } \\
\cline { 4 - 7 } & & & & & Upper bound & Lower bound \\
\hline Thalamus & 0.187 & 0.802 & 0.321 & 0.152 & 0.078 & 0.297 \\
R insula & 0.821 & 0.653 & 0.857 & 0.697 & 0.718 & 0.925 \\
L insula & 0.835 & 0.734 & 0.750 & 0.758 & 0.735 & 0.936 \\
\hline
\end{tabular}

Abbreviations: AUC, area under the curve; HC, healthy control; HZ, herpes zoster; L, left; R, right; ROC, receiver operating characteristic.

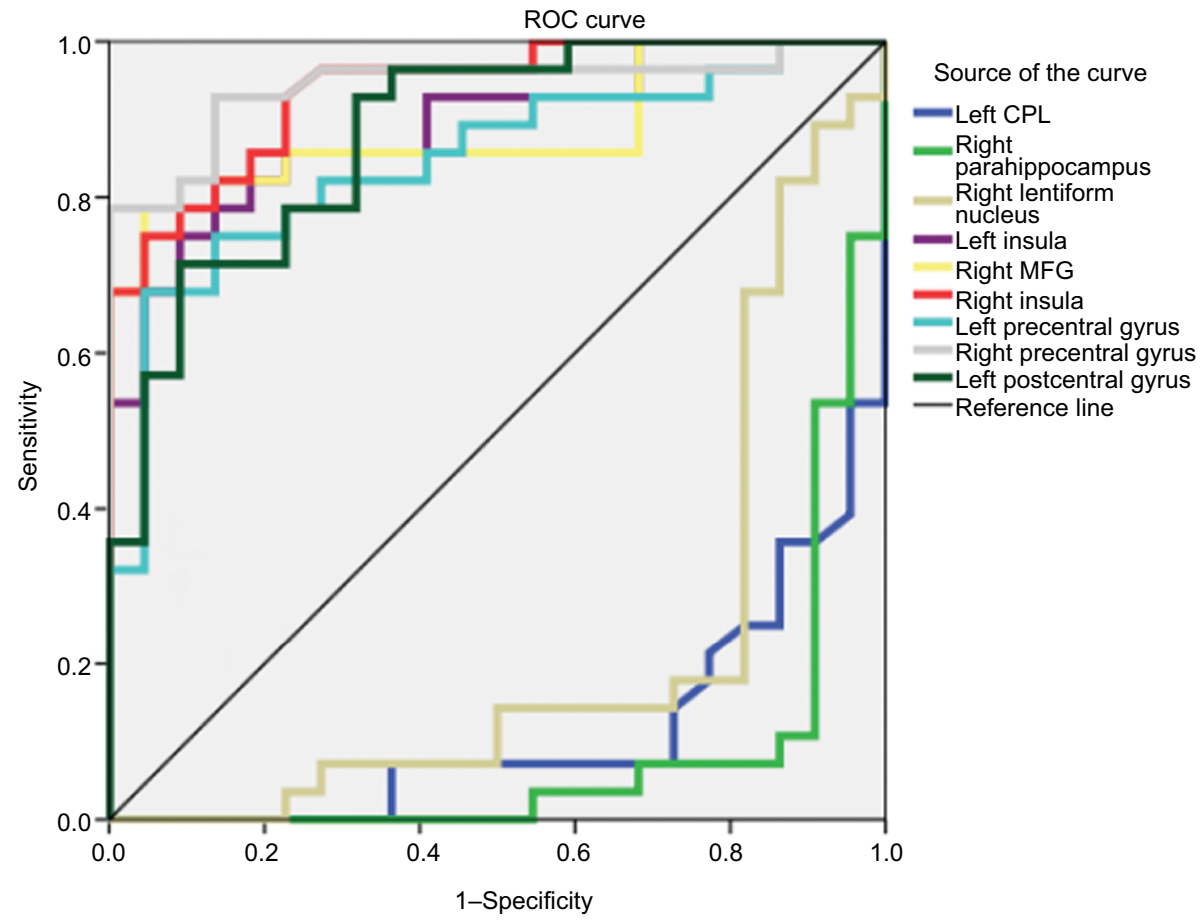

Figure 7 ROC curves of GMV in the altered regions for distinguishing PHN patients from HCs.

Abbreviations: CPL, cerebellar posterior lobe; GMV, gray matter volume; HCs, healthy controls; MFG, middle frontal gyrus; PHN, postherpetic neuralgia; ROC, receiver operating characteristic.

Table 6 ROC analysis for differentiating the PHN and HC groups

\begin{tabular}{|c|c|c|c|c|c|c|}
\hline \multirow[t]{2}{*}{ Brain region } & \multirow[t]{2}{*}{ AUC } & \multirow{2}{*}{$\begin{array}{l}\text { Cutoff } \\
\text { point }\end{array}$} & \multirow[t]{2}{*}{ Sensitivity (\%) } & \multirow[t]{2}{*}{ Specificity (\%) } & \multicolumn{2}{|l|}{$95 \% \mathrm{Cl}$} \\
\hline & & & & & Upper bound & Lower bound \\
\hline L CPL & 0.113 & 0.993 & 0.071 & 0.273 & 0.023 & 0.202 \\
\hline R Parahippocampus & $0.08 I$ & 0.755 & 0.107 & 0.091 & 0.000 & 0.167 \\
\hline $\mathrm{R}$ lentiform nucleus & 0.218 & 0.787 & 0.179 & 0.192 & 0.075 & 0.360 \\
\hline$L$ insula & 0.890 & 0.718 & 0.75 & 0.909 & 0.801 & 0.978 \\
\hline R MFG & 0.885 & 0.6745 & 0.786 & 0.955 & 0.789 & 0.981 \\
\hline$R$ insula & 0.938 & 0.637 & 0.75 & 0.955 & 0.876 & 0.999 \\
\hline L precentral gyrus & 0.847 & 0.439 & 0.75 & 0.864 & 0.739 & 0.956 \\
\hline R precentral gyrus & 0.942 & 0.266 & 0.929 & 0.864 & 0.875 & 1.000 \\
\hline L postcentral gyrus & $0.88 I$ & 0.706 & 0.714 & 0.909 & 0.789 & 0.974 \\
\hline
\end{tabular}

Abbreviations: AUC, area under the curve; CPL, cerebellar posterior lobe; HC, healthy control; L, left; MFG, middle frontal gyrus; PHN, postherpetic neuralgia; R, right; ROC, receiver operating characteristic.

tral mechanism of PHN. Further ROC analysis showed that decreased GMV in the bilateral insula, bilateral precentral gyrus, right MFG, and left postcentral gyrus corresponded to greater AUCs (it is accepted that AUC has a significant diagnostic when the value is $>0.6),{ }^{23}$ suggesting that these regions could serve as markers to separate individuals with PHN from healthy subjects. PHN patients exhibited greater GMV in the left cerebellar tonsil, culmen, and left lentiform 


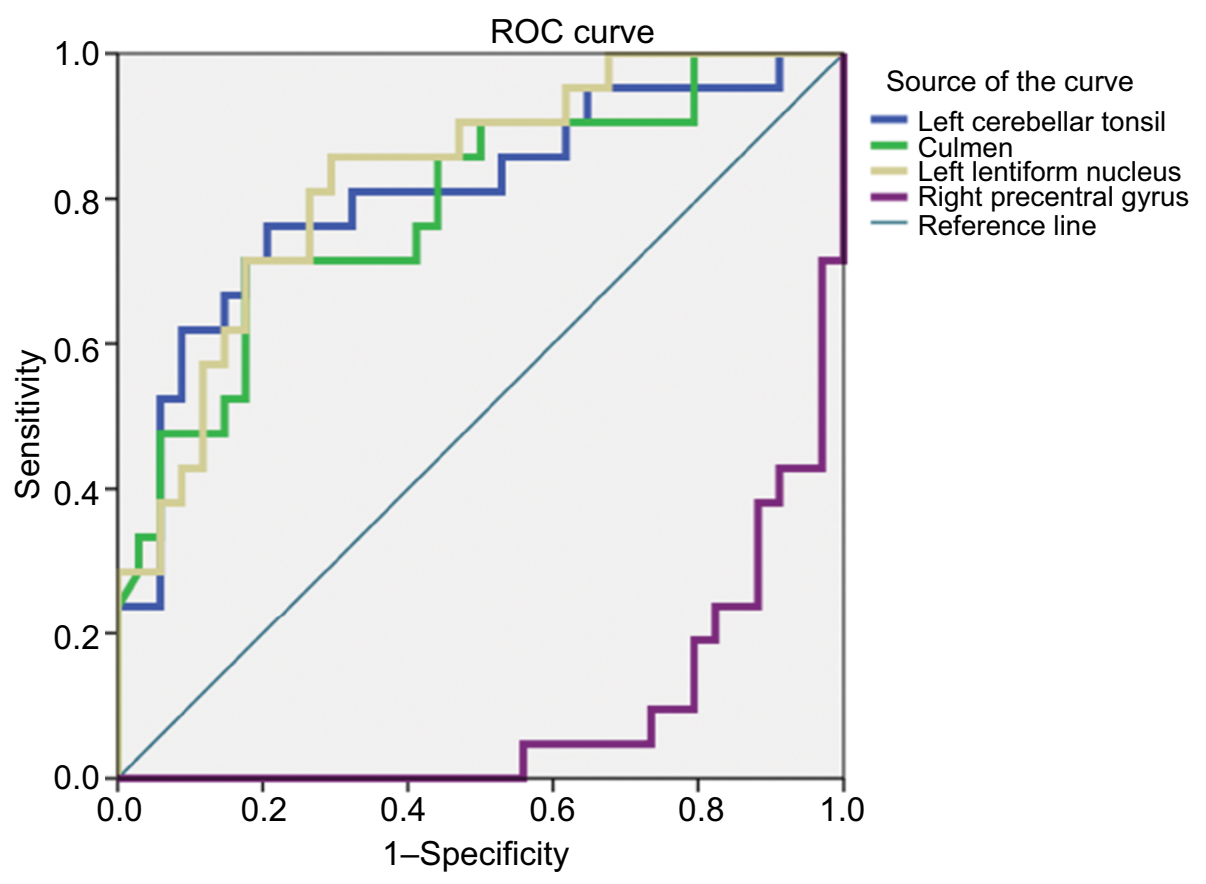

Figure 8 ROC curves of GMV in the altered regions for distinguishing PHN patients from $\mathrm{HZ}$ patients.

Abbreviations: GMV, gray matter volume; $\mathrm{HZ}$, herpes zoster; $\mathrm{PHN}$, postherpetic neuralgia; $\mathrm{ROC}$, receiver operating characteristic.

Table 7 ROC analysis for differentiating the PHN and $\mathrm{HZ}$ groups

\begin{tabular}{|l|l|l|l|l|l|l|}
\hline Brain region & AUC & Cutoff & Sensitivity (\%) & Specificity (\%) & \multicolumn{2}{l|}{$\mathbf{9 5 \%}$ Cl } \\
\cline { 6 - 8 } & & point & & & Upper bound & Lower bound \\
\hline L cerebellar tonsil & 0.805 & 0.426 & 0.762 & 0.794 & 0.679 & 0.932 \\
Culmen & 0.788 & 1.188 & 0.714 & 0.824 & 0.661 & 0.914 \\
L lentiform nucleus & 0.826 & -0.471 & 0.857 & 0.706 & 0.716 & 0.937 \\
R precentral gyrus & 0.091 & -0.063 & 0.095 & 0.206 & 0.016 & 0.166 \\
\hline
\end{tabular}

Abbreviations: AUC, area under the curve; HZ, herpes zoster; L, left; PHN, postherpetic neuralgia; R, right; ROC, receiver operating characteristic.

nucleus than $\mathrm{HZ}$ patients. These regions corresponded to greater AUCs in the ROC analysis, suggesting that these regions may be involved in $\mathrm{HZ}$ transformation to PHN.

Previous studies have demonstrated the important role of the insular lobe in pain-related diseases, such as migraine, ${ }^{24}$ low back pain, ${ }^{25}$ and trigeminal neuralgia. ${ }^{26}$ The results showed that decreased GMV in the bilateral insular lobes together with higher AUCs is common in $\mathrm{HZ}$ and PHN patients, suggesting the essential role of the insula in pain. Zhang et a ${ }^{15}$ showed similar results that $\mathrm{PHN}$ patients exhibited significantly decreased DKI parameters in the bilateral insular lobes compared with HCs. The insula mainly accepts direct projections from the medial thalamus and is part of the medial pain sensory system. In fact, neuroimaging studies have demonstrated the critical role of the insula in pain pathways and its close relationship with the perceived intensity of nociceptive stimuli. ${ }^{27}$ Not only does the insula directly receive pain sensation information and can act to relieve pain, but it can also affect subsequent decisions and behaviors. ${ }^{28}$ In addition, neuropathic pain causes biochemical alterations of dopamine receptors and muscarinic receptors in the insula. ${ }^{29}$ Therefore, the abnormal perception of nociceptive stimulation caused by decreased GMV in the insula may be an important mechanism of hyperalgesia. Moreover, the left insula had a negative correlation with pain duration in PHN patients. All these findings suggest that changes in GMV in the insula are specific to pain and may be involved in pain progression, specifically that more severe structural damage to the insula causes extended pain duration. These findings prove that the insula is an indispensable brain region that could play a fundamental role in pain modulation.

The thalamus is the most important sensory conduction relay station for all senses except the sense of smell and plays a pivotal role in cortical pain signal input regulation and cor- 
tical excitability control..$^{30}$ Increased GMV in the thalamus might reflect central sensitization in pain diseases resulting from more pain signal input. fMRI studies have shown increased cerebral blood flow and ReHo values in the thalamus are associated with pain intensity in PHN patients, ${ }^{9}, 13$ and growing evidence has shown that the thalamus plays a vital role in the production and maintenance of neuropathic pain. ${ }^{31}$ For example, neuropathic pain is associated with anatomical changes, biochemical changes, ${ }^{32}$ and perfusion changes in the thalamus. Our study demonstrated that the increase in GMV in the thalamus is correlated with VAS scores in HZ patients, possibly indicating that the thalamus is sensitive to pain and reflects pain intensity. However, some studies reported atrophy of the thalamus GM caused by pain. ${ }^{33}$ The inclusion of samples with different durations of pain may be related to these conflicting results, which could also be a reason why no abnormalities of the thalamus GMV were found in PHN patients.

The VBM analysis results revealed that the $\mathrm{HZ}$ and $\mathrm{PHN}$ patients showed abnormal GM in several brain regions, including the bilateral insula, thalamus, right MFG, left posterior central gyrus, and cerebellum, compared with HCs. These areas overlap with the "pain matrix", which is defined as regions that are activated in response to increasing levels of pain, ${ }^{34}$ mainly including the somatosensory area, supplementary motor area, insular lobes, thalamus, forebrain, and cingulate gyrus. ${ }^{34,35}$ Table 3 and Figure 2 show that, in addition to regions of the pain matrix, the right parahippocampus, right lentiform nucleus, and bilateral precentral gyrus are implicated in brains of PHN patients. Among them, the lentiform nucleus, which has rich connections with the attentional sensorimotor and limbic emotion processing network, is a crucial component of the striatum. ${ }^{13}$ As the striatum makes up a significant part of reward, hedonic, and emotional circuitries, ${ }^{36,37}$ increased GMV in the lentiform nucleus could explain the emotional abnormities in PHN patients. These results suggest not only that PHN affects cerebral pain perception but also that $\mathrm{PHN}$ pathogenesis involves more complicated, variational central mechanisms, which may contribute to its poor treatment outcomes.

The parahippocampus, as part of the limbic system, plays an important role in memory function. Increased GMV of the parahippocampus was positively correlated with VAS scores in our results, indicating that bad experiences caused by pain may form memories, and the formation of these memories further worsens the pain experience, which may be one of the reasons behind refractory pain in PHN patients. Furthermore, amygdala sits at the tip of the hippocampus and there are evidences that patients with sustained anxiety or treated depression may show increased volume in amygdala. ${ }^{38,39} \mathrm{We}$ are not exactly clear how PHN patients are treated during shingles, although we scanned them before treatment. It may be another reason for the increased GMV of parahippocampus. However, our results are contrary to those of previous studies of PHN. For example, Zhang Yi et al ${ }^{15}$ reported that DKI parameters were decreased in the parahippocampal gyrus, and Chen et $\mathrm{a}^{14}$ used DTI and found that fractional anisotropy was reduced in the parahippocampus. Zhang Yue et $\mathrm{al}^{40}$ related $\mathrm{PHN}$ pain to the small-world properties of brain functional networks and detected significant decreases in the integrated nodal efficiency in the bilateral parahippocampal gyrus. From an anatomical point of view, the increased GMV may be related to neuronal proliferation, an increased number of synapses, or vascular changes, while many researchers agree that it is a type of compensatory response of adaptive nature. The result of GMV alteration in parahippocampus that contradicts some of the previous studies may be because of the differences of individuals. Nevertheless, it is possible that microstructural abnormalities in the parahippocampus might correspond to the memory aspect of the pain reaction in PHN, and antidepressants treatment may be one of the reasons as well.

As expected, the $\mathrm{HZ}$ and PHN brains exhibited different morphological changes. Significantly increased GMV was revealed in the left cerebellar tonsil, culmen, and left lentiform nucleus of PHN patients compared with $\mathrm{HZ}$ patients. These regions showed greater AUCs in the ROC analysis, indicating that they could distinguish PHN from HZ, and abnormalities in these regions of the brain are risk factors for HZ patients to develop PHN. The PHN brain also showed decreased GMV in the right precentral gyrus, and these results are in agreement with the longitudinal study results of Cao et al. ${ }^{41}$

Recently, more studies have identified that the cerebellum is involved in pain processing and regulation. fMRI studies have shown abnormal activity of the cerebellum in PHN brains. ${ }^{36,42}$ Restuccia et al ${ }^{43}$ indicated that different types of pain activate different subregions of the cerebellum. Our results show that changes in the GMV of the CPL were positively correlated with VAS scores, which could explain the close relationship between the cerebellum and pain. The cerebellum and the precentral gyrus are both important motor regulatory centers. Alterations of GMV in these regions may be related to pain epiphenomena, such as the suppression of daily movement or pain-evoked movements. ${ }^{44}$ Due to the long-term effects of allodynia, patients with PHN often 
adjust their posture throughout the day to prevent contact with clothes to avoid tactile allodynia. Interestingly, the cerebellum is closely related to mental disorders and is also linked to depression. ${ }^{45}$ This may be one of the reasons why PHN patients have a high risk of developing depression. Under the long-time affect of the negative emotion, neuropathic pain is more difficult to be cured and more likely to develop PHN. Cerebellum also involves emotional regulation and cognition, which may lead the transformation of simple neuropathic pain to more complex neuralgia with multiple factors after its anomaly. Therefore, the cerebellum is a multi-effect integrator that involves pain processing, motor regulation, emotional regulation, and cognitive function. ${ }^{46}$

\section{Limitations}

There are some limitations to this study. First, the small sample size is a common problem in this and other PHN studies. Because all patients were recruited from the Pain Department, our investigation lacks patients with lower pain intensity levels (VAS $<5$ ). The range of pain duration among the PHN patients is also a limitation (mean of 3 months). Patients with a wider range of pain intensities and longer pain duration should be recruited to verify these results in future studies. Second, the emotional statuses of the subjects, which may affect the brain microstructure, were not scaled in this study. Although no obvious emotional symptoms were observed among the patients, our results showed abnormalities in emotion-related regions. Third, it remains unknown whether alterations in GMV are the cause of pain or the result of pain. Considering that the adult human brain may change its structure in response to environmental demands, ${ }^{47}$ a thorough longitudinal study is needed to explore whether morphological changes can be reversed when nociceptive inputs cease or when the disease is adequately treated. Also, whether the side of the herpetic lesion correlates with the side of the brain involvement is worth investigating.

\section{Conclusion}

Both HZ and PHN could extensively influence the brain structure. Larger GMV alterations in PHN brains indicate the complexity of the central mechanism - PHN is not only connected with pain-related regions but also with regions related to emotion, memory, and behavior. The GMVs of the bilateral insula, bilateral precentral gyrus, right MFG, and left postcentral gyrus could be imaging markers for differentiating PHN patients from healthy individuals. Additionally, the cerebellum, lentiform nucleus, and precentral gyrus may play essential roles in the transition of $\mathrm{HZ}$ to PHN.

\section{Acknowledgments}

This study was supported by grants from the Nature Science Foundation of Jiangxi Province (grant no. 2015ZBAB205021 and grant no. 20191042), Jiangxi Province Education Department Support Program (grant no. GJJ160128), and the Health Commission of Jiangxi Province (grant no. 20181BAB205028).

\section{Author contributions}

$\mathrm{JL}, \mathrm{LG}, \mathrm{FZ}$, and JJ designed the study. JL, SH, QH, LG, and DZ acquired the data. FZ, JJ, LG, and JL processed the neuroimaging data and performed statistical analyses. JL, LG, QH, SH, XZ, DZ, FZ, and JJ wrote the initial draft. All authors contributed to data analysis, drafting and revising the article, gave final approval of the version to be published, and agree to be accountable for all aspects of the work.

\section{Disclosure}

The authors report no conflicts of interest in this work.

\section{References}

1. Johnson RW, Rice AS. Clinical practice. Postherpetic neuralgia. NEngl J Med. 2014;371(16):1526-1533.

2. Pickering G, Leplege A. Herpes zoster pain, postherpetic neuralgia, and quality of life in the elderly. Pain Pract. 2011;11(4):397-402.

3. Wang JS, Bao JJ, Wei X, Du WQ. A clinical investigation on postherpetic neuralgia (PHN). Chin J Pain Med. 2011;17:198-200.

4. Baron R, Binder A, Wasner G. Neuropathic pain: diagnosis, pathophysiological mechanisms, and treatment. Lancet Neurol. 2010;9(8):807-819.

5. Peng WW, Guo XL, Jin QQ, et al. Biological mechanism of post-herpetic neuralgia: evidence from multiple patho-psychophysiological measures. Eur J Pain. 2017;21(5):827-842.

6. Baliki MN, Chialvo DR, Geha PY, et al. Chronic pain and the emotional brain: specific brain activity associated with spontaneous fluctuations of intensity of chronic back pain. J Neurosci. 2006;26(47):12165-12173.

7. Davis KD, Moayedi M. Central mechanisms of pain revealed through functional and structural MRI. J Neuroimmune Pharmacol. 2013;8(3):518-534.

8. Cao S, Song G, Zhang Y, et al. Abnormal local brain activity beyond the pain matrix in postherpetic neuralgia patients: a resting-state functional MRI study. Pain Physician. 2017;20(2):E303-E314.

9. Liang HW, Xiao LZ, Qiu YH. The comparative study of cerebral functional in different phase of herpes zoster by ReHO fMRI. Chin J Pain Med. 2014;20(10):717-721.

10. Liang HW, Xiao LZ. Comparison of low frequency fluctuation amplitude for cerebral function in two phases of trunk herpes zoster. Chin J Rehabil. 2015;2:106-108.

11. Liao X, Chen FY, Tao W, et al. Functional magnetic resonance study of brain basal activity changes in patients with postherpetic neuralgia. Pro Biochemi and Biophysics. 2015;10:947-954.

12. Jiang J, Gu L, Bao D, et al. Altered homotopic connectivity in postherpetic neuralgia: a resting state fMRI study. J Pain Res. 2016;9:877-886.

13. Liu J, Hao Y, Du M, et al. Quantitative cerebral blood flow mapping and functional connectivity of postherpetic neuralgia pain: a perfusion fMRI study. Pain. 2013;154(1):110-118.

14. Chen F, Chen F, Shang Z, et al. White matter microstructure degenerates in patients with postherpetic neuralgia. Neurosci Lett. 2017;656:152-157.

15. Zhang Y, Yu T, Qin B, Li Y, Song G, Yu B. Microstructural abnormalities in gray matter of patients with postherpetic neuralgia: a diffusional kurtosis imaging study. Pain Physician. 2016;19(4):E601-E611. 
16. Wang SD, Lu Y, Zhao W, et al. Observation of morphological changes of brain gray matter volume in patients with postherpetic neuralgia using VBM-DARTEL method. J Pract Radiol. 2017;33(9):1337-1340.

17. Head H, Campbell AW, Kennedy PG. The pathology of herpes zoster and its bearing on sensory localisation. Rev Med Virol. 1997;7(3):131-143.

18. Watson CP, Morshead C, Van der Kooy D, Deck J, Evans RJ. Post-herpetic neuralgia: post-mortem analysis of a case. Pain. 1988;34(2):129-138.

19. Hong $\mathrm{S}, \mathrm{Gu} \mathrm{L}, \mathrm{Zhou} \mathrm{F}$, et al. Altered functional connectivity density in patients with herpes zoster and postherpetic neuralgia. J Pain Res. 2018;11:881-888.

20. Kelly AM. Does the clinically significant difference in visual analog scale pain scores vary with gender, age, or cause of pain? Acad Emerg Med. 1998;5(11):1086-1090.

21. Bijur PE, Silver W, Gallagher EJ. Reliability of the visual analog scale for measurement of acute pain. Acad Emerg Med. 2001;8(12):1153-1157.

22. Ivo R, Nicklas A, Dargel J, et al. Brain structural and psychometric alterations in chronic low back pain. Eur Spine J. 2013;22(9):1958-1964.

23. Zhou F, Gu L, Hong S, et al. Altered low-frequency oscillation amplitude of resting state-fMRI in patients with discogenic low-back and leg pain. J Pain Res. 2018;11:165-176.

24. Zheng SN, Wang YJ, Hu YK. Analysis of gray matter abnormalities based on voxel-based morphometry in migraine patients: meta-analysis. Chin J Med Imaging Technol. 2016;32(10):1480-1484.

25. Mao CP, Yang QX, Tang J. Brain gray matter abnormalities revealed by voxel-based morphometry in patients with chronic low back pain. $J$ South Med Univ. 2016;36(8):1041-1047.

26. Jiang YM, Huang JQ, Li ZF. Abnormalities of gray matter volume in patients with idiopathic trigeminal neuralgia: a voxel-based morphometry study. Chin J Med Imaging Technol. 2015;31(6):826-830.

27. Dun WH, Yang J, Yang L, et al. Abnormal structure and functional connectivity of the anterior insula at pain-free periovulation is associated with perceived pain during menstruation. Brain Imaging Behav. 2017:11(6):1787-1795.

28. Brooks JCW, Tracey I. The insula: a multidimensional integration site for pain. Pain. 2007;128(1):1-2.

29. Ferrier J, Bayet-Robert M, Dalmann R, et al. Cholinergic neurotransmission in the posterior insular cortex is altered in preclinical models of neuropathic pain: key role of muscarinic M2 receptors in donepezilinduced antinociception. J Neurosci. 2015;35(50):16418-16430.

30. Borsook D. Neurological diseases and pain. Brain. 2012;135(Pt 2):320-344.

31. Wu Y, Wang LJ, Yun LN. Research progress of brain functional magnetic resonance imaging in pertherpetic neuralgia patients. Chin J Pain Med. 2017;23(5):371-375. Chinese.

32. Gustin SM, Wrigley PJ, Youssef AM, et al. Thalamic activity and biochemical changes in individuals with neuropathic pain after spinal cord injury. Pain. 2014;155(5):1027-1036.
33. Apkarian AV, Sosa $\mathrm{Y}$, Sonty $\mathrm{S}$, et al. Chronic back pain is associated with decreased prefrontal and thalamic gray matter density. J Neurosci. 2004;24(46):10410-10415.

34. Garcia-Larrea L, Peyron R. Pain matrices and neuropathic pain matrices: a review. Pain. 2013;154(Suppl. 1):S29-S43.

35. Legrain V, Iannetti GD, Plaghki L, Mouraux A. The pain matrix reloaded: a salience detection system for the body. Prog Neurobiol. 2011;93(1):111-124

36. Geha PY, Baliki MN, Chialvo DR, Harden RN, Paice JA, Apkarian AV. Brain activity for spontaneous pain of postherpetic neuralgia and its modulation by lidocaine patch therapy. Pain. 2007;128(1-2):88-100.

37. Geha PY, Baliki MN, Wang X, Harden RN, Paice JA, Apkarian AV. Brain dynamics for perception of tactile allodynia (touch-induced pain) in postherpetic neuralgia. Pain. 2008;138(3):641-656.

38. Hamilton JP, Siemer M, Gotlib IH. Amygdala volume in major depressive disorder: a meta-analysis of magnetic resonance imaging studies. Mol Psychiatry. 2008;13(11):993-1000.

39. Vytal KE, Overstreet C, Charney DR, Robinson OJ, Grillon C. Sustained anxiety increases amygdala-dorsomedial prefrontal coupling: a mechanism for maintaining an anxious state in healthy adults. $J$ Psychiatry Neurosci. 2014;39(5):321-329.

40. Zhang Y, Liu J, Li L, et al. A study on small-world brain functional networks altered by postherpetic neuralgia. Magn Reson Imaging. 2014;32(4):359-365.

41. Cao S, Qin B, Zhang Y, et al. Herpes zoster chronification to postherpetic neuralgia induces brain activity and grey matter volume change. Am J Transl Res. 2018;10(1):184-199.

42. Cao S, Li Y, Deng W, et al. Local brain activity differences between herpes zoster and postherpetic neuralgia patients: a resting-state functional MRI study. Pain Physician. 2017;20(5):E687-E699.

43. Restuccia D, Della Marca G, Valeriani M, Leggio MG, Molinari M. Cerebellar damage impairs detection of somatosensory input changes. A somatosensory mismatch-negativity study. Brain. 2007;130(Pt 1):276-287.

44. Apkarian AV, Bushnell MC, Treede RD, Zubieta JK. Human brain mechanisms of pain perception and regulation in health and disease. Eur J Pain. 2005;9(4):463-484.

45. Moulton EA, Schmahmann JD, Becerra L, Borsook D. The cerebellum and pain: passive integrator or active participator? Brain Res Rev. 2010;65(1):14-27.

46. Buckner RL. The cerebellum and cognitive function: 25 years of insight from anatomy and neuroimaging. Neuron. 2013;80(3):807-815.

47. Draganski B, Gaser C, Busch V, Schuierer G, Bogdahn U, May A. Neuroplasticity: changes in grey matter induced by training. Nature. 2004;427(6972):311-312
Journal of Pain Research

\section{Publish your work in this journal}

The Journal of Pain Research is an international, peer reviewed, open access, online journal that welcomes laboratory and clinical findings in the fields of pain research and the prevention and management of pain. Original research, reviews, symposium reports, hypothesis formation and commentaries are all considered for publication.

\section{Dovepress}

The manuscript management system is completely online and includes a very quick and fair peer-review system, which is all easy to use. Visit http://www.dovepress.com/testimonials.php to read real quotes from published authors. 